\title{
Roadside Infrastructure Placement for Information Dissemination in Urban ITS Based on a Probabilistic Model
}

\author{
Bo Xie, Geming Xia, Yingwen Chen, and Ming Xu \\ Dept. of Network Engineering, Computer School, \\ National University of Defense Technology, \\ Changsha, China \\ \{xiebo, xuming\}@nudt.edu.cn, xiageming@126.com, \\ csywchen@gmail.com
}

\begin{abstract}
Information dissemination is an important application in VANETs for traffic safety and efficiency. In urban area, roadside infrastructure nodes can be deployed for information dissemination. However, it is inefficient and uneconomical to cover the whole urban area. How to find the optimal locations to place DPs is a research problem. Some works on this issue have to collect accurate trajectories of all the vehicles, which is not practical in the real environment. In this paper, we propose a novel approach for DPs placement in grid road networks without knowing trajectories. Based on the analysis of path number between two intersections, a probabilistic model is proposed to get the trajectories estimation of vehicles. The theoretical optimal algorithm (OA) and two heuristic algorithms (called KP-G and GA) are developed for the problem. Simulation results reveal that GA is scalable and has the highest coverage ratio on average.
\end{abstract}

\section{$1 \quad$ Introduction}

Information dissemination based on Vehicular Ad hoc Networks (VANETs) is intended to the support traffic safety and efficiency, as well as services for drivers [1-3]. In this paper, we deal with information dissemination from roadside infrastructure to passing vehicles, tackling the specific issue of deploying an intelligent transport system infrastructure that efficiently achieves the dissemination goal. For example, transport department can disseminate some traffic news to vehicles. We refer to the vehicles who have received the disseminated information as informed vehicles. Our goal is to maximize the number of informed vehicles. In other words, we aim at maximizing the coverage ratio of information dissemination.

In principle, an information dissemination system could leverage both vehicle-tovehicle (V2V) and vehicles-to-infrastructure (V2I) communications. When only a few of roadside units (RSUs) are deployed, V2V communications could enable data sharing thus increasing the coverage ratio of information dissemination. However, the gain achieved through V2V communication strictly depends on the network topology 
and the particular cooperation paradigm, and it is difficult to evaluate in the general case. In this paper, we analyze the problem of optimally placing infrastructure nodes (e.g. IEEE 802.11 access points or RSUs) only considering V2I communications.

We refer to the infrastructure nodes as Dissemination Points (DPs). A DP serves for the vehicles that pass through the dissemination range of the DP. In other words, the vehicles who pass through a DP could be served (i.e., covered). However, it is difficult, in terms of infrastructure cost, to cover all roads with a large number of DPs, especially during the rollout of ITS. In our approach, DPs are placed at intersections which prove to be much better locations than road segments for DPs deployment in [4]. We could describe this problem as follows: in a given urban area which has $N$ intersections with a limited number of $k$ DPs ( $k \leq N$ ), what is the best deployment strategy to maximize the coverage ratio of information dissemination.

We could model the problem as a Maximum Coverage Problem (MCP). However, traditional approaches of MCP may not be suitable for this problem for three reasons. First, the DPs deployed in the area neither have to necessarily form a connected network, nor provide a continuous coverage of the road topology. Second, vehicles move among these different DPs rather than be stationary. Third, vehicles may cross more than one intersection, thus they may be served by more than one DPs. The problem presented in this paper differs from traditional problems.

This problem could be solved through heuristic algorithms if we could get the accurate trajectory of every vehicle as in [4]. However, the drivers may not be willing to share their trajectories for privacy concerns. As a consequence, it is not practical to collect every vehicle's accurate trajectory for decision-making. Moreover, because the trajectories of all vehicles and the traffic pattern may change from time to time, the placement based on trajectories may not be stable. It means that the optimal placement based on a certain set of trajectories may not be optimal in another set of trajectories. Meanwhile, in real communication environments, it could not assure that a vehicle could receive the information from a DP when it passes the DP. We introduce a parameter $p s$ to represent the probability that a vehicle could successfully receive information from a DP. The probability $p s$ is a feature of a wireless link and may affect the deployment of DPs. Our scheme deploys DPs at the most appropriated locations based on $p s$ rather than improving $p s$.

In this paper, we propose a novel approach to solve the problem without using vehicles' accurate trajectories. Instead of vehicles' accurate trajectories, we only need the road network topology, and vehicles' origin points and destination points. Note that in a certain observation time, most vehicles except taxis have specific origin points and destination points in one journey. Based on the analysis on the statistical or historical data, we could get the distributions of the numbers of vehicles at origin points and destination points. Then the trajectories estimation of vehicles could be derived. Then, we propose a theoretical optimal algorithm (OA) and two heuristic algorithms (called KP-G and GA) for the problem.

The remainder of this paper is organized as follows. Section 2 reviews the previous work. Section 3 presents a probabilistic model, and proposes an optimal algorithm as well as two heuristic algorithms. Performance evaluations are presented in Section 4. Section 5 concludes this paper. 


\section{Related Work}

Wireless access point or base station placement is a well known research topic. In the context of sensor networks, several studies have considered the optimal node placement schemes which are always NP-hard. To tackle such complexity, several heuristics have been proposed to find sub-optimal solutions [5, 6] in presence of stationary nodes.

In VANETs and mesh networks, there are several studies on roadside units (RSUs) placement problem. Lochert et al. [7] have tackled the problem of sparse roadside units placement for data aggregation but not information dissemination. Sun et al. [8] propose cost-efficient RSUs deployment scheme for short-time certificate updating. In this scheme, OnBoard Units (OBUs) in any place could communicate with RSUs in certain driving time, and the extra overhead time used for adjusting routes to update short-time certificates is small. Pan et al. [9] address the problem of optimally placing one or multiple gateways in both 1-D and 2-D vehicular networks to minimize the average number of hops from APs. They also give some analytical results for finding the optimal placement of multiple gateways in 2-D vehicular grid networks and discuss how to minimize the total power consumption. Abdrabou et al. [10] present an analytical framework to statistically estimate the maximum packet delivery delay from a vehicle to an RSU for a low density VANET via vehicle-to-vehicle communications. Aoun et al. [11] propose a polynomial time near-optimal algorithm which recursively computes minimum weighted Dominating Sets (DS), while consistently preserving QoS requirements across iterations. Trullols et al. [4] have done the closest work to ours. They propose three heuristic deployment algorithms MCP-g, MCP-sz and KP-P for information dissemination in intelligent transportation systems. However, their heuristic algorithms need the accurate trajectory of every vehicle. The difference between our work and [4] is that we could make approximate optimal DPs placement only based on road network topology and vehicles' origin points and destination points. We also consider the probability of vehicles served.

\section{$3 \quad$ Probabilistic Model and Placing Algorithms}

\subsection{Problem Statement}

We consider an urban grid road network. As in many Chinese cities, the road networks are very regular which could be mapped into grid road networks. Literature [4] reveals that intersections prove to be much better locations than road segments for DPs deployment. Thus, we also place DPs at intersections. We assume that each DP cover only one intersection, which means that only vehicles cross it could be served (i.e., covered). Our goal is to place the $k$ DPs for maximizing the number of informed vehicles. However, we would not need vehicle's accurate trajectories for two reasons. As first important, vehicles may not share their trajectories for privacy concerns, and it is also impossible and unpractical to collect those sensitive individual data. On the 
other hand, the placements based on trajectories are not stable due to trajectories may change time to time. It is not flexible to deploy DPs in real world.

We propose a probabilistic model based on the distributions of the origin points and destination points of vehicles in an urban environment. As we know, most vehicles except taxis have specific origin points and destination points in one journey. For example, during 7:00am and 8:00am, they drive from their home to offices. Then they park their vehicles near their offices. The residence is the origin point, and the parks near offices are the destination points. While the path from origin point to destination point is uncertain for different reasons. For example, they could choose the path according to the real time traffic, or according to their favor, or by GPSbased navigation systems, and so on. We study the probability of vehicles crossing each intersection by random path selection.

\subsection{Number of Paths}

In a given grid road network, the number of paths between two intersections is needed to be calculated. We assume that vehicles always select the shortest paths. Traditional approaches search the shortest paths using graph theory such as Dijkstra algorithm. However, it is not sufficient for grid networks due to so many paths with the same shortest distance. As shown in Fig.1, we assume the length of each segment is the same.

Let $\operatorname{FP}\left(\left(i_{1}, j_{1}\right)\left(i_{2}, j_{2}\right)\right)$ denote the number of paths between $\left(i_{1}, j_{1}\right)$ and $\left(i_{2}, j_{2}\right)$. To reduce the dimension of FP, we use a integer $x$ to represent the intersection $(i, j)$ in $m \times n$ grid road network, where $x=(i-1) \cdot n+j$. Therefore, $\operatorname{FP}\left(\left(i_{1}, j_{1}\right)\left(i_{1}, j_{1}\right)\right)$ could be denoted as $f p\left(x_{1}, x_{2}\right)$.

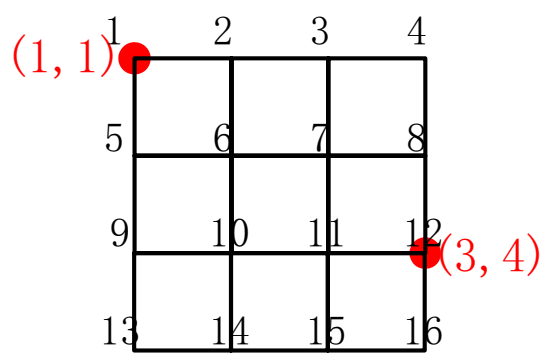

Fig. 1. $4 \times 4$ Grid Road Network

$f p\left(x_{1}, x_{2}\right)$ could be calculated as Eq.(1).

$$
f p\left(x_{1}, x_{2}\right)=F P\left(\left(i_{1}, j_{1}\right)\left(i_{2}, j_{2}\right)\right)=F(\Delta i, \Delta j)=\frac{(\Delta i+\Delta j)}{\Delta i ! \Delta j !}
$$

In Eq.(1), $\Delta i=\left|i_{1}-i_{2}\right|$, and $\Delta j=\left|j_{1}-j_{2}\right|$. For example, $f p(1,12)=\operatorname{FP}((1,1)(3,4))$ $=\mathrm{F}(2,3)=10$. 


\subsection{Origin Points and Destination Points}

The origin points and destination points will be any places on the road segment; however, we could map these points into the intersections. For example, as shown in Fig. 2, a vehicle named A starts from point a where located at north of the road segment. The circle line figures out the dissemination range of each DP. The origin point of vehicle A is out range of any DPs. According to the traffic rule, vehicles should run on the right side, therefore, we regard the origin point of vehicle A as intersection $(2,2)$. It is the similar for destination points. If a vehicle's destination point is out range of any DP, we regard the last intersection which it has crossed as its destination point. We use matrices $G$ and $D$ to denote the distributions of the numbers of vehicles at origin points and destination points.

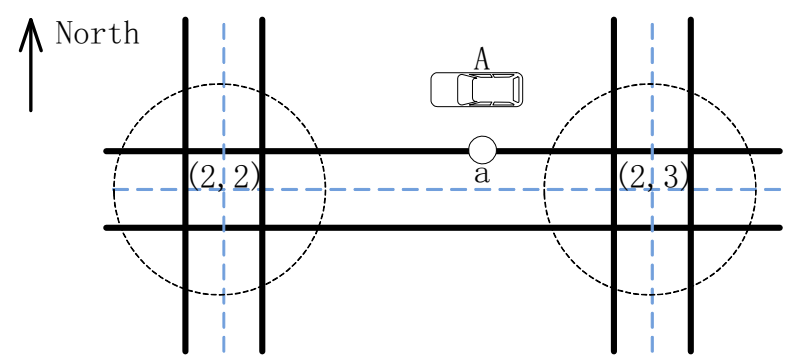

Fig. 2. Mapping Origin Point into Intersection

\subsection{Probabilistic Model and Algorithms}

In the $m \times n$ grid network, let $\left(i_{k}, j_{k}\right)$ denote the original address of intersection $x_{k}$. Here, $1 \leq i_{k} \leq m, 1 \leq j_{k} \leq n$ and $x_{k}=\left(i_{k}-1\right) \cdot n+j_{k}$. We introduce several probabilities as listed Table 1 .

We use Coverage Ratio $(C R)$ to evaluate the final performance of the algorithms. $C R$ means the proportion of the number of vehicles served by DPs to the number of total vehicles during the observation time. We could use Eq.(2) to compute $C R$.

Table 1. Probability Symbols

\begin{tabular}{l|l}
\hline Symbol & Description \\
\hline$p s_{x}$ & The probability that a vehicle is served when it crosses $x$ \\
\hline$p a_{x}$ & The probability that a vehicle has once appeared at $x$ \\
\hline$p g_{x}$ & The probability that a vehicle starts from $x$ \\
\hline$p d_{x}$ & The probability that a vehicle disappears at $x$ \\
\hline$p o d_{x_{o} x_{d}}$ & The probability that a vehicle is start from $x_{o}$ and disappears at $x_{d}$ \\
\hline$p p_{x}$ & $\begin{array}{l}\text { The probability that a vehicle passes through the intersection } x \text { but } \\
\text { neither starts from nor disappears at } x\end{array}$ \\
\hline$p f_{c}\left(x_{1}, \ldots, x_{c}\right)$ & $\begin{array}{l}\text { The probability that a vehicle passes through these } c \text { intersections, } \\
c>1\end{array}$ \\
\hline
\end{tabular}




$$
C R=\frac{\left|\bigcup_{i=1}^{i=k} V_{x_{i}} \cdot p s_{x_{i}}\right|}{N V T}<\sum_{i=1}^{i=k} p a_{x_{i}} \cdot p s_{x_{i}}
$$

In Eq.(2), $V_{x_{i}}$ means the set of vehicles that cross $x_{i}$, and NVT means the total number of vehicles during the observation time. Therefore, $\mid \bigcup_{i=1}^{i=k} V_{x_{i}} \cdot p s_{x_{i}}$ Imeans the total number of vehicles that have been served by one or more of DPs which are placed at these $k$ intersections. However, it is quite difficult to compute the accurate $C R$. It is obvious that $C R$ is fewer than $\sum_{i=1}^{i=k} p a_{x_{i}} \cdot p s_{x_{i}}$ which could be used as approximate value and obtained with less computation. According to the above analysis, we propose an optimal algorithm OA, and two heuristic algorithms KP-G and GA. The optimal algorithm OA could only be used in small scale situations due to its high computational complexity.

The optimal algorithm OA and heuristic algorithm KP-G are listed as following, respectively.

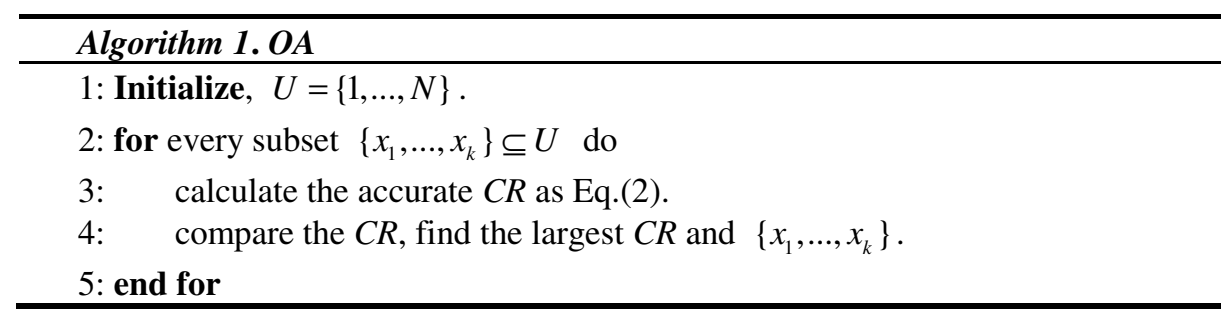

\section{Algorithm 2. KP-G}

1: Initialize, $v=\{0\}$.

2: for every intersection $x_{i}$ do

3: $\quad v_{i}=p a_{x_{i}} \cdot p s_{x_{i}}$.

\section{4: end for}

5: sort $v$ in descending order, select the first $k$ intersections

Obviously, $p f_{k}<p f_{k-1}<\ldots<p f_{2}<p a, \quad p f_{2}$ plays a more important role in Eq.(2) than other $p f_{c}$ with $c>2$. In large grid road networks, $p f_{c}$ are relatively small. Therefore, in GA, we use $p f_{2}$ to approach the accurate $C R$. It will lose some performance comparing with $\mathrm{OA}$, but it has quite low computational complexity.

We use $k=3$ to explain how to compute $C R$ with $p f_{2}$ as Eq.(3). 


$$
\begin{aligned}
& C R=\frac{\left|\bigcup_{i=1}^{i=k} V_{x_{i}} \cdot p s_{x_{i}}\right|}{N V T} \\
& \approx\left(p a_{x_{1}}-p f_{2}\left(x_{1}, x_{2}\right)-p f_{2}\left(x_{1}, x_{3}\right)\right) \cdot p s_{x_{1}} \\
& +\left(p a_{x_{2}}-p f_{2}\left(x_{1}, x_{2}\right)-p f_{2}\left(x_{2}, x_{3}\right)\right) \cdot p s_{x_{2}} \\
& +\left(p a_{x_{3}}-p f_{2}\left(x_{1}, x_{3}\right)-p f_{2}\left(x_{2}, x_{3}\right)\right) \cdot p s_{x_{3}} \\
& +p f_{2}\left(x_{1}, x_{2}\right) \cdot\left(1-\left(1-p s_{x_{1}}\right) \cdot\left(1-p s_{x_{2}}\right)\right) \\
& +p f_{2}\left(x_{1}, x_{3}\right) \cdot\left(1-\left(1-p s_{x_{1}}\right) \cdot\left(1-p s_{x_{3}}\right)\right) \\
& +p f_{2}\left(x_{2}, x_{3}\right) \cdot\left(1-\left(1-p s_{x_{2}}\right) \cdot\left(1-p s_{x_{3}}\right)\right)
\end{aligned}
$$

The heuristic algorithm GA is listed as following.

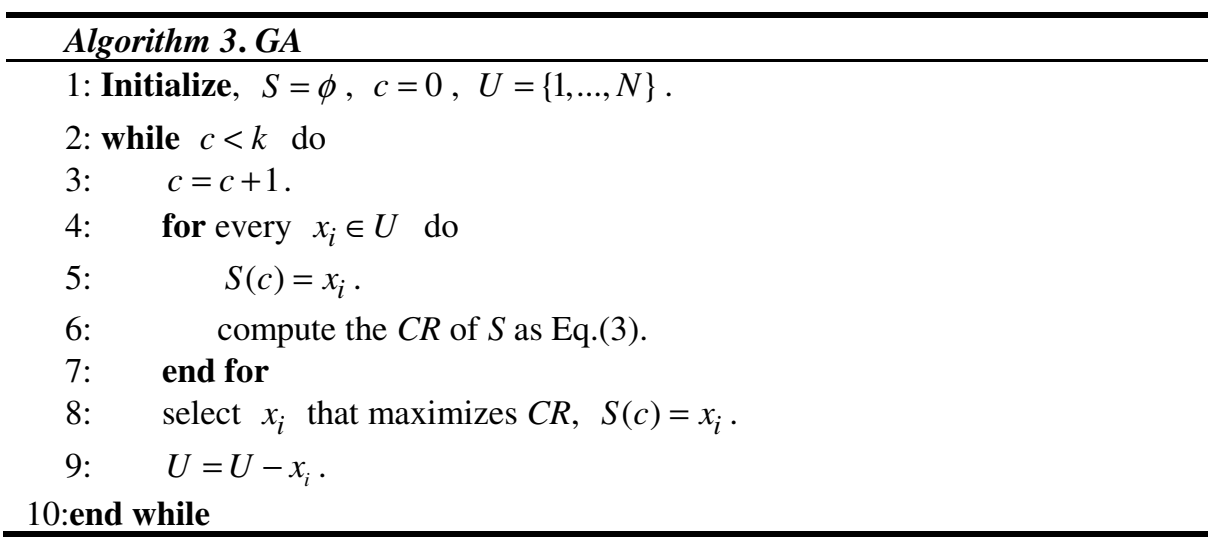

\section{Performance Evaluation}

In this section, we conduct simulations to evaluate the performance of the proposed algorithms. Note that, we do not concern ourselves with low-level issues in wireless communications.

In [4], a heuristic algorithm KP-P is proposed based on the knowledge of the number of vehicles crossing each intersection. KP-P sorts the intersections in descending order by their crossing vehicles, and selects the first $k$ intersections to place DPs. Using the trajectories generated by our simulator, we could implement KP-P. Therefore, the results of KP-P could be regarded as the simulation results of our KP-G. The $C R$ of GA, KP-G, OA, and KP-P are evaluated in this section. Therefore, we compare the four algorithms in the following subsections.

\subsection{Small Scale Scenarios}

In this subsection, we use small $m, n, k$, and we compare the four algorithms. Due to computational complexity, $\mathrm{OA}$ is computationally feasible only for small values of $k$ in very small scale scenarios. We use $4 \times 4$ grid road network as shown in Fig. 1 . When $k>4$, the computational complexity is too high to obtain the optimal solution. We give the $C R$ of OA with $k=2,3,4$. We use the same $p s_{x}$ for every intersection. 
As shown in Fig. 3, we could get the following conclusions. First, both simulation results of OA and KP-G are excellently agree with the analytical results. It demonstrates the accuracy of our model. Second, GA is much better than KP-G and $\mathrm{KP}-\mathrm{P}$, and even has the same performance as OA. For a given $C R$, GA needs fewer DPs, and for a certain number of DPs, GA could achieve higher $C R$. However, with the decline of $p s$, the performance benefit of GA becomes more unremarkable, and the overall CRs of all algorithms become smaller. In real environment, we always attend to increase the probability $p s$, therefore GA is much more useful than other algorithms.

We then study the detailed placements of the four algorithms. For example, Table 2 shows the placements with $p s=1$. The integers represent the intersection sequence numbers as shown in Fig. 1. When $k=2$, the placements of the four algorithms are respectively $\{11,6\},\{11,10\},\{10,7\},\{6,11\}$. When $k=4$, the placements are respectively $\{11,6,8,9\},\{11,10,7,6\},\{11,7,10,6\},\{4,7,10,13\}$. With the increment of $k$, we only need to add additional points into the original sets for GA, KP-G, KP-P, whereas we have to change all the points of OA. In other words, GA, KP-G, KP-P could yield incremental placements, whereas OA has to compute the results for different $k$. The difference between the placements of KP-G and KP-P is not remarkable. Note that, $C R$ of GA reaches 1 for $k=6$, whereas KP-G and KP-P both need $8 \mathrm{DPs}$ for $C R=1$. For GA, the placements listed in Table 2 is not unique for $k=8$. The last two intersections could be replaced by any other intersections.

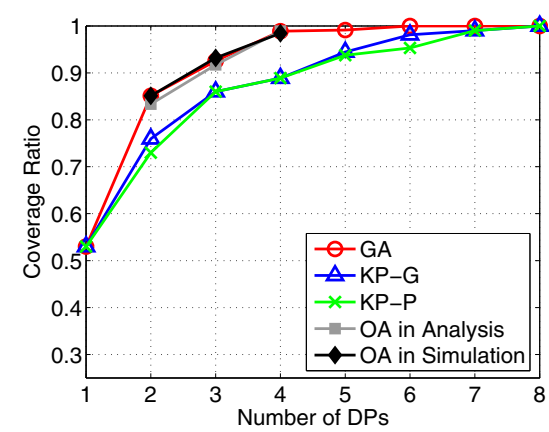

(a) $p s=1$

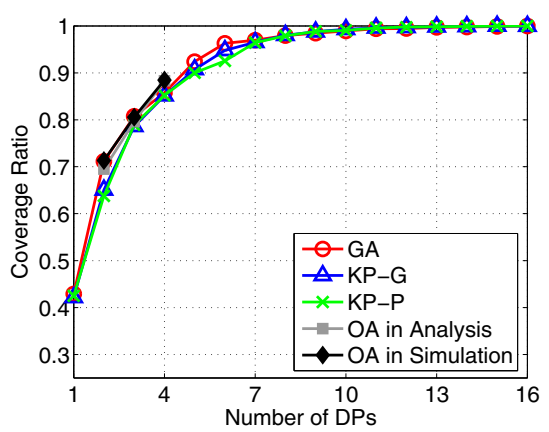

(b) $p s=0.8$

Fig. 3. $C R$ versus the number of DPs deployed, for $4 \times 4$ grid road network

Table 2. Placement of Every Algorithm

\begin{tabular}{l|l}
\hline Algorithm & Placement \\
\hline GA & $\{11,6,8,9,3,14,16,1\}$ \\
\hline KP-G & $\{11,10,7,6,15,2,14,8\}$ \\
\hline KP-P & $\{11,7,10,6,14,15,2,3\}$ \\
\hline OA & $\{6,11\},\{6,11,16\},\{4,7,10,13\}$ \\
\hline
\end{tabular}

\subsection{Large Scale Scenarios}

For a large grid network of $6 \times 9$, there are $N=54$ intersections. We study more DPs as $k=27$ which is half of $N$. Because OA could not be solved within acceptable 
time even when $k=2$ in this large network, we only compare GA with KP-G and KP-P. We generate a pair of random $6 \times 9$ matrices of $G$ and $D$ which are referred to as $G_{2}$ and $D_{2}$, and generate another symmetrical pair of matrices as $G_{3}$ and $D_{3}$. In $G_{3}$ and $D_{3}$, all vehicles start from and also disappear at the borders of the grid. It is an extreme special case.

There are similar conclusions with that in small scale scenarios. Furthermore, from Fig. 4, we could find that different $G$ and $D$ cause different performances. However, GA is still better than KP-G and KP-P. In Fig. 4(b), CRs of KP-G and KP-P have remarkable difference for some $k$ due to its extreme special $G_{3}$ and $D_{3}$. Whatever the different $G$ and $D$ are, the benefit of GA is very remarkable, especially with $3<k<13$.

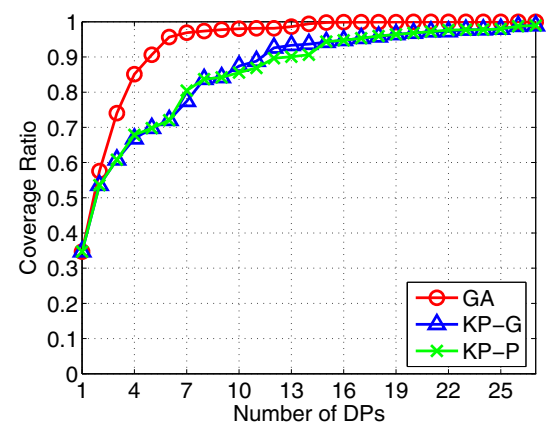

(a) $G_{2}, D_{2}$

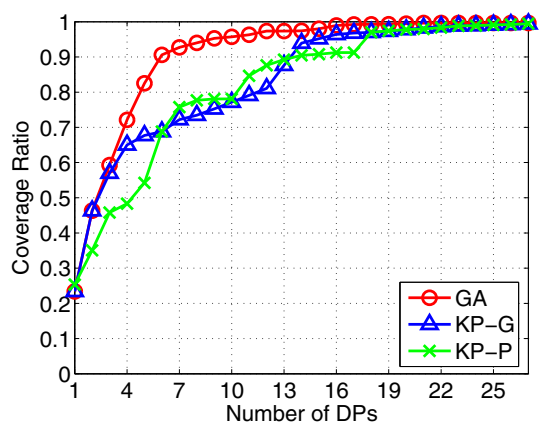

(b) $G_{3}, D_{3}$

Fig. 4. $C R$ versus the number of DPs deployed, for $6 \times 9$ grid road network, $p s=1$

\section{Conclusion}

In this paper, we proposed a novel maximum coverage approach for disseminating information to vehicles in intelligent system in urban area without using vehicles' trajectories. We proposed a probabilistic model based on the distributions of the numbers of vehicles at origin points and destination points to get the trajectories estimation of the vehicles. We applied this model to compute the coverage ratio. Next, an optimal algorithm OA and two heuristic algorithms KP-G and GA were proposed. It was not practical to adopt OA due to high computational complexity. Our results proved that GA had better performance than the other algorithms in most of scenarios. However, we still remarked that in different scenarios with different parameters and conditions, it was better to compare the algorithms and choose the best one.

In fact, the road networks of many cities are not regular grid. We could still map the road networks into grids through some mechanisms which will be our future work. Drivers may choose paths with the different probabilities, thus other path selection models will also be considered in our future work. 
Acknowledgment. This research is partially supported by the National Science Foundation of China under Grant No. 61070211, No. 61272485, No. 61070201 and No. 61003304; and Hunan Provincial Natural Science Foundation of China under grants No. 09JJ4034.

\section{References}

1. Dikaiakos, M.D., Iqbal, S., Nadeem, T., Iftode, L.: Vitp: an information transfer protocol for vehicular computing. In: Proceedings of the 2nd ACM International Workshop on Vehicular Ad Hoc Networks, VANET 2005, pp. 30-39. ACM, New York (2005)

2. Mak, T.K., Laberteaux, K.P., Sengupta, R.: Amulti-channel vanet providing concurrent safety and commercial services. In: Proceedings of the 2nd ACM International Workshop on Vehicular Ad Hoc Networks, VANET 2005, pp. 1-9. ACM, New York (2005)

3. Yang, X., Liu, L., Vaidya, N., Zhao, F.: A vehicle-to-vehicle communication protocol for cooperative collision warning. In: The First Annual International Conference on Mobile and Ubiquitous Systems: Networking and Services, MOBIQUITOUS 2004, pp. 114-123 (August 2004)

4. Trullols, O., Fiore, M., Casetti, C., Chiasserini, C., Barcelo Ordinas, J.: Planning roadside infrastructure for information dissemination in intelligent transportation systems. Computer Communications 33(4), 432-442 (2010)

5. Arkin, E.M., Efrat, A., Mitchell, J.S., Polishchuk, V., Ramasubramanian, S., Sankararaman, S., Taheri, J.: Data transmission and base-station placement for optimizing the lifetime of wireless sensor networks. Ad Hoc Networks (in press)

6. Capone, A., Cesana, M., Donno, D.D., Filippini, I.: Deploying multiple interconnected gateways in heterogeneous wireless sensor networks: An optimization approach. Computer Communications 33(10), 1151-1161 (2010)

7. Lochert, C., Scheuermann, B., Wewetzer, C., Luebke, A., Mauve, M.: Data aggregation and roadside unit placement for a vanet traffic information system. In: Proceedings of the Fifth ACM International Workshop on VehiculAr Inter-NETworking, VANET 2008, pp. 58-65. ACM, New York (2008)

8. Sun, Y., Lin, X., Lu, R., Shen, X., Su, J.: Roadside units deployment for efficient shorttime certificate updating in vanets. In: 2010 IEEE International Conference on Communications (ICC), pp. 1-5 (May 2010)

9. Li, P., Huang, X., Fang, Y., Lin, P.: Optimal placement of gateways in vehicular networks. IEEE Transactions on Vehicular Technology 56(6), 3421-3430 (2007)

10. Abdrabou, A., Zhuang, W.: Probabilistic delay control and road side unit placement for vehicular ad hoc networks with disrupted connectivity. IEEE Journal on Selected Areas in Communications 29(1), 129-139 (2011)

11. Aoun, B., Boutaba, R., Iraqi, Y., Kenward, G.: Gateway placement optimization in wireless mesh networks with qos constraints. IEEE Journal on Selected Areas in Communications 24(11), 2127-2136 (2006) 